\title{
Auf der Suche nach der Innovation: Grenzgänger zwischen Wissenschaft und Wirtschaft
}

\begin{abstract}
Die Dynamik technischer Neuerungen sowie die Entwicklung auf den Arbeitsmärkten erfordern Modifikationen in den tradierten Berufsrollen. Das gilt auch für Wissenschaftlerinnen und Wissenschaftler im Bereich der öffentlichen Forschung. Zunehmend gefragt ist der Typus des Wissenschaftsunternehmers, der in idealer Weise wissenschaftliche und ökonomische Bezüge in einer Person verbindet. Zugleich belegen Studien über Wissenschaftler, die Unternehmen gegründet haben, dass Änderungen oder Neuschöpfungen von Berufsrollen im akademischen Alltagsgeschäft ein sehr schwieriges und langwieriges Unterfangen sind. Ein Wechsel zu neuen beruflichen Identitäten gelingt im deutschen Wissenschaftssystem daher eher selten, ist aber - wie der Beitrag aufzeigt - unter spezifischen Bedingungen möglich.
\end{abstract}

\section{Diversifikation von Rollen- erwartungen}

Das Tätigkeitsspektrum von Wissenschaftlerinnen und Wissenschaftlern ${ }^{1}$ ist längst nicht mehr auf ihre Kernaufgaben in Lehre und Forschung eingrenzbar. Sie müssen nicht erst seit heute auch in Gremien Entscheidungen fällen, begutachten, Forschungsmittel einwerben, den wissenschaftlichen Nachwuchs ausbilden und mit Stellen versorgen, Institute führen, Wissenschaftsverwaltungen und Wirtschaft beraten, sich der Öffentlichkeit mitteilen und das Wissen möglichst auch in ökonomische Verwertungszusammenhänge transferieren, sei es in Kooperation mit Unternehmen der gewerblichen Wirtschaft oder direkt als Spin-off, als akademische Ausgründung. Idealtypisch findet sich die Vorstellung einer zunehmenden Erweiterung der Rolle des Wissenschaftlers mit ökonomischen Bezügen in dem seit geraumer Zeit im internationalen Kontext genutzten Begriff des „Wissenschaftsunternehmers" oder auch "Wissenschaftsentrepreneur" wieder (Meyer 2003). Gemeint sind hier Forscher, die parallel zur wissenschaftlichen Praxis auch in Unternehmensgründungen oder in anderer Weise in unternehmerische Aktivitäten eingebunden sind. Sie stehen vor der Herausforderung, wissenschaftliche und wirtschaftliche Relevanzen und Belange in einer Berufsrolle zu integrieren. Ob das gelingt und unter welchen Bedingungen es gelingen kann, ist nicht nur für die berufliche Identität dieser Akteure von gro- ßer Bedeutung. Die Diversifizierung von Rollenerwartungen ist nämlich der Ausgangspunkt ganz verschiedener Diagnosen darüber, in welcher gesellschaftlichen Lage sich Wissenschaft heute befindet und welche wissenschaftspolitischen Schlussfolgerungen daraus gezogen werden müssen (Simon et al. 2010). Die Ausdehnung und Ausweitung des Tätigkeitsspektrums von Wissenschaftlern auf Bereiche, die vermeintlich nicht zu den Kerntätigkeiten gehören, wird einerseits gerne als Indiz für eine generelle „Entgrenzung“ gesellschaftlicher Funktions- und Zuständigkeitsbereiche gedeutet („Blurring of Boundaries“). Im Falle des Wissenschaftsunternehmers sehen Autoren aus den USA schon seit Jahren eine neue Fähigkeit entwickelt, bei der Aspekte der Wissens- und Erkenntniserweiterung mit „Profit-Motiven“ (Etzkowitz 1998) kombiniert werden. Andere Autoren sprechen dagegen von einer generellen „Ökonomisierung der Wissenschaft“ im Rahmen einer Wissensökonomie und sehen Belege für einen „Academic Capitalism" (Slaughter/Leslie 1997). Wiederum andere Autoren deuten die Diversifikation wissenschaftlicher Tätigkeit eher als eine generelle Entprofessionalisierung. Für das Fischen in fremden Gewässern würden Wissenschaftler über keine hinreichenden Kompetenzen verfügen und schon deshalb in Konkurrenz zu anderen Berufsgruppen geraten (Musselin 2007). Schlimmer noch: Dabei würden die Forscher die Fähigkeit verlieren, ihre eigenen Belange der wissenschaftlichen Arbeit angemessen weiterzuentwickeln (Münch 2007).

Neben diesen wissenschaftssoziologischen Thematisierungsarten ist die Frage nach den Möglichkeiten und Grenzen einer
Rollendiversifikation des Wissenschaftlers aber auch für politische Gestaltungswünsche von Bedeutung. In jüngster Zeit lassen sich nämlich mehrere Versuche der Wissenschafts- und Hochschulpolitik beobachten, die allesamt auf die Veränderung der akademischen Sozialisation und Berufsrolle des Wissenschaftlers abzielen. So hat der Bolognaprozess neben einer Harmonisierung und Modularisierung von Studiengängen auch zum Ziel, eine stärkere Berufsorientierung in die akademischen Ausbildungsgänge zu implementieren (Gülker et al. 2009). Begleitet werden diese erweiterten Ziele von einer Reihe neu eingeführter betriebswirtschaftlich orientierter

\footnotetext{
Mit „Wissenschaftler", "Unternehmer" und "Wissenschaftsunternehmer" sind Berufsrollen gemeint, die sich gegenüber der Kategorie Geschlecht neutral verhalten. Es sind stets Männer und Frauen damit angesprochen, selbst wenn in unserer Erhebung und vermutlich auch darüber hinaus, rein quantitativ betrachtet, sehr wenige Wissenschaftsunternehmerinnen vertreten sind.
}

Marc Torka, Dr. phil, ist wissenschaftlicher Mitarbeiter der Forschungsgruppe Wissenschaftspolitik am Wissenschaftszentrum Berlin für Sozialforschung (WZB). Arbeitsschwerpunkte: Wissenschafts-, Professionssoziologie und Innovations forschung. e-mail:torka@wzb.eu Andreas Knie, Prof. Dr., lehrt Soziologie an der TU Berlin und ist Geschäftsführer des Innovationszentrums Mobilität und gesellschaftlicher Wandel (InnoZ) GmbH. Arbeitsschwerpunkte: Mobilitäts-, Wissenschafts- und Innovationsforschung. e-mail: knie@wzb.eu 
Managementinstrumente. Professoren werden im Rahmen der neuen W-Besoldungsstruktur Angebote unterbreitet, durch eine Intensivierung und Ausdehnung ihrer wissenschaftlichen Arbeit eine Steigerung des Gehalts zu erreichen. In turnusmäßigen Evaluationen wird ein wissenschaftliches Verständnis gepflegt, bei dem anhand von Outputleistungen die Qualität gemessen und die ökonomische Affinität dabei bewusst gesucht wird („,value for money"). Schließlich finden sich zahlreiche Fördermaßnahmen, die Kooperationen mit der Industrie und den Unternehmen der gewerblichen Wirtschaft unterstützen. Mit einer Reihe prominenter Förderprogramme soll die Verbindung wissenschaftlicher und wirtschaftlicher Orientierungen intensiviert werden. Ob es der vom Forschungsministerium initiierte Wettbewerb um sogenannte Spitzencluster ist oder die vom Wirtschaftsministerium geförderten Maßnahmen zur Entstehung und nachhaltigen Absicherung von Gründungskulturen an Hochschulen (EXIST) - in allen diesen staatlichen Programmen wird eine engere Verzahnung wissenschaftlicher Einrichtungen mit unternehmerischem Denken gesucht (Egeln et al. 2010).

Sowohl die sozialwissenschaftlichen Beschreibungen der aktuellen Lage der Wissenschaft sowie die wissenschaftspolitischen Bestrebungen legen also nahe, dass ökonomisches Denken und Handeln für die Wissenschaft bedeutsamer geworden sind. Im Folgenden soll der Frage nachgegangen werden, ob sich diese Verschiebungen auch in geänderten und erweiterten Vorstellungen von der Berufsrolle des Wissenschaftlers niederschlagen und ob möglicherweise der „Wissenschaftsunternehmer" hier einen integrierenden Ansatz darstellen könnte. Abschließend werden die dabei aufgeworfenen Fragen hinsichtlich der wissenschaftspolitischen Realisierbarkeit diskutiert.

\section{Wissenschaftsunternehmer und Innovation}

Bereits im Begriff der Innovation verschmelzen wirtschaftliche und wissenschaftliche Bezüge, denn Neuerung kann sich gleichermaßen auf Ideen und produktartige Erfindungen beziehen. Die Idee, Wissenschaft und Wirtschaft stärker miteinander zu verbinden, ist aber nicht neu. Bereits im Zuge der Industrialisierung und der dabei einsetzenden Erkenntnis, dass neue Produkte und Produktionsweisen zunehmend Wissen als Ressource benötigen, entstand eine Vielzahl technischer Ausbildungsstellen, die mehr und mehr auf wissenschaftliche Grundlagen gestellt wurden. Die im letzten Drittel des 19. Jahrhunderts gegründeten Technischen Hochschulen waren der institutionalisierte Wille, wirtschaftliches Wettbewerbsdenken auf der Basis eines akademischen Wissenskanons zu entwickeln. Selbst die wissenschaftlich höchst reputierliche Max-Planck-Gesellschaft wurde als Kaiser-Wilhelm-Gesellschaft mit dem expliziten Ziel gegründet, im wirtschaftlichen Wettbewerb der Nationen besser bestehen zu können, indem sich Grundlagenforschung in neuer Form und mithilfe privaten Kapitels entwickeln konnte. Parallel entstanden Wirtschaftszweige, die unmittelbar auf wissenschaftlichen Erkenntnissen fußten. Diese „science based industries" (Rammert 1988), wie die Chemie- und Pharmabranche, verbinden bis heute systematische wissenschaftliche Erkenntnisarbeit mit unternehmerischer Geschäftslogik innerhalb multinationaler Konzernstrukturen. In den 1930er und 40er Jahre rückte öffentlich finanzierte und akademisch orientierte Wissenschaft im Interesse der militärischen Weltkriegsplanungen so eng mit Unternehmen zusammen, dass von äußerst stabilen wissenschaftlich-wirtschaftlichen-militärischen Komplexen gesprochen wurde. Spektakulärstes Beispiel ist das in Los Alamos organisierte „Manhatten-Project“, in dem eine Reihe späterer Nobelpreisträger sehr konkret an der Entwicklung der ersten Atombombe arbeitete. Obwohl in den 1940er und 50er Jahren unter dem Eindruck des Schreckens der Weltkriege offiziell eine Entflechtung wirtschaftlicher Interessen und wissenschaftlicher Forschung deklariert wurde (Bush 1945), blieben die Verbindungen faktisch immer eng. Verändert haben sich lediglich die Formen der Beziehungen. Mal wurden umfangreiche Grundlagenforschungskapazitäten während der 1960er Jahre innerhalb der Industrie selbst aufgebaut, dann wiederum organisierte sich die Zusammenarbeit mehr in Formen loser Kooperation oder seit wenigen Jahren auch in neuen Hybridgebilden, wie sie unter dem Label „industry on campus“ von Großkonzer- nen wie IBM oder Microsoft an etablierten Universitätsstandorten wie Oxford oder Cambridge erprobt werden (Knie/BraunThürmann 2008).

Seit den späten 1970er Jahren ist dabei die Vorstellung präsent, dass Innovationen nicht mehr in einem Kaskadenmodell (,erst wissenschaftliche Erkenntnis, dann die Produkte') entstehen, sondern durch eine Gleichzeitigkeit wissenschaftlicher und produktorientierter Denk- und Vorgehensweisen, die gleichsam rekursive Schleifen durchlaufen. Immer stärker schiebt sich dabei der Begriff der „Validierung" in den Vordergrund. Gemeint ist dabei der Versuch, durch wirtschaftliche Experimente die Forschungsergebnisse „sozial robust“ zu machen und dann auch im Wettbewerb industrieller Verwertungsstrategien angemessen zu platzieren (Braun-Thürmann et al. 2010).

Die Idee, Wissenschaft stärker an ökonomische Belange anschlussfähig zu halten, findet aber nicht nur in der Wirtschaft, sondern auch aus der Wissenschaft heraus Unterstützung. Neben der zeitlich befristeten und in den Erfolgsaussichten sehr ungewissen öffentlichen Forschungsfinanzierung bieten Industriegelder erweiterte Optionen. Dies gilt für die Unterstützung wissenschaftlicher Arbeit genauso wie auch für die weitere Berufsorientierung. Denn in berufsbiografischer Hinsicht bleibt eine wissenschaftliche Karriere äußerst riskant und Alternativen sind zwingend notwendig.

Schließlich deutet auch vieles auf habituelle Gemeinsamkeiten zwischen dem Sozialtypus des Wissenschaftlers und des Unternehmers hin, die zu einem neuen Typus des Wissenschaftsunternehmers zusammenfließen könnten. Schon bei Schumpeter zielt unternehmerisches Handeln auf Neuerung durch das „Erkennen und Durchsetzen neuer Möglichkeiten auf wirtschaftlichem Gebiet" (Schumpeter 1928, S. 151) ab. Zentrale Voraussetzung hierbei ist eine besonderes „Mindset“, das auch in der wissenschaftlichen Forschung verbreitet ist: „Freimut zu riskanter Entscheidung mit dem Anspruch auf rationale Verlässlichkeit“ (Loer 2006, S. 7). Im Grunde gibt es also genügend Gemeinsamkeiten, die auf den ersten Blick für eine Überlappung von Interesse, Orientierungen und Identitäten sprechen könnten. 


\section{Berufsverständnis von Wissenschaftsunter- nehmern}

Um der These einer möglichen Konvergenz zwischen den Berufsrollen von Wissenschaftlern und Unternehmern nachzugehen, ist am Wissenschaftszentrum Berlin für Sozialforschung (WZB) eine Reihe von empirischen Untersuchungen über das Berufsverständnis von Wissenschaftlern durchgeführt worden ${ }^{2}$. In mehr als 100 berufsbiografischen Interviews wurden Beteiligte aller Hierarchiestufen und Disziplinbereiche (insbesondere aber Biotechnologie sowie Informations- und Kommunikationstechnik) über Ausgründungsmotive und -prozesse sowie die beruflichen (Um-)Orientierungen befragt und die Antworten entlang einer grundsätzlichen Frage analysiert: Inwiefern liegen unterschiedliche Anforderungen des Wirtschafts- bzw. Wissenschaftssystems vor und inwieweit gelingt es, diese in einer Person dauerhaft zu vereinbaren? Kann man darüber Einblick in die Bedingungen der Genese einer neuen Berufsrolle „Wissenschaftsunternehmer" gewinnen?3

Im Folgenden werden zentrale Ergebnisse dieser Studien vorgestellt. Sie werfen Licht auf die Schwierigkeit, in einem für das Innovationssystem sensiblen Bereich neue Berufsrollen zu konstruieren und zu etablieren.

\subsection{BERUFSBIOGRAFISCHER ENTSCHEIDUNGSZWANG}

Ein erstes Ergebnis bei der Auswertung der Interviews ist, dass ausgerechnet die Personengruppe, die in ihrer Berufsbiografie sowohl Aspekte einer wissenschaftlichen wie auch eines unternehmerischen Engagements (Professor und Unternehmen) erfolgreich vereinen, auf Befragen nahezu ausnahmslos für eine frühzeitige Entscheidung der Karriereorientierung plädiert. Man sollte sich frühzeitig über seine berufliche Identität klar werden und hier die notwendigen biografischen Entscheidungen treffen - so unisono die Meinung der Befragten. Dieser Rat zu einer sehr frühen Entscheidung ist nicht normativer Natur und hat auch nichts mit einer Innovationsfeindlichkeit der deutschen Wissenschaft oder dem fehlenden
Ausgründungswillen von Wissenschaftlern zu tun, sondern ist rein strukturell begründet. Diese Einsicht stammt aus einer sehr grundlegenden Erfahrung, die praktisch alle Ausgründenden in Deutschland machen: Es fehlt vordergründig die Zeit, die unterschiedlichen Anforderungsprofile zu integrieren. Die Produktionsrhythmen von operativer unternehmerischer Arbeit und wissenschaftlicher Erkenntnisproduktion sind trotz einiger bemerkenswerter Affinitäten in einem Bereich entscheidend unterschiedlich: Sofern Wissenschaftler während eines unternehmerischen Engagements weiterhin ihre Chance auf eine wissenschaftliche Karriere aufrechterhalten wollen, müssen sie wissenschaftlich relevante Publikationen nachweisen. Die gegenwärtig beobachtbare Verschärfung einer Outputorientierung und die Standardisierung von Altersgrenzen verstärken diesen Zwang zum Publizieren zusätzlich. Der Arbeitsnachweis in der Publikationsliste gilt wiederum als wichtige Zugangsvoraussetzung für die akademischen Qualifizierungsprozesse, deren Einhaltung streng beobachtet wird und die als Voraussetzung für eine akademische Karriere gelten: Die Aussagen der Beteiligten aller Hierarchiestufen waren in diesem Sinne sehr eindeutig: entweder gelingt die Habilitation oder die Aussicht auf eine Professur ist trotz unternehmerischer Tätigkeit praktisch zu Ende. Die „Peers“, die Fachkollegen, verlangen insbesondere auch in den naturwissenschaftlichen Disziplinen den Respekt für die eigenen Qualitätskriterien, und die sind eindeutig wissenschaftsintern ausgelegt: Artikel in referierten Zeitschriften, Qualifizierungsnachweise durch Promotion und Habilitation. Ausgründungen sind so lange akzeptiert, so lange sie die wissenschaftlichen Referenzen nicht bedrohen. Ist der Sprung auf eine Professur gelungen, dann bleibt ebenfalls wenig Zeit, um neben den eingangs erwähnten multiplen Rollenanforderungen auch noch unternehmerisch tätig zu sein. Gleichzeitig ein Unternehmen zu führen und einen Lehrstuhl zu organisieren, das gelingt nur wenigen. Die Regel im deutschen Wissenschaftssystem lautet daher mehrheitlich: Ganz oder gar nicht! Interessanterweise treten diese scharfen Demarkationslinien auch und gerade in den Disziplinen auf, die im internationalen Kontext gerne als flexibel und durchlässig gelten (Torka/ Borcherding 2008).
Und dennoch findet man auch vor diesem berufsbiografischen Erfahrungshintergrund immer wieder Konstellationen, bei denen Hochschullehrer Ausgründungen initiieren oder sich an solchen beteiligen: Professoren betreiben beispielsweise Ausgründungen ,nach und neben' einer wissenschaftlichen Karriere und übernehmen dabei eine anregende, unterstützende und beratende Funktion. Eine lange und stabile wissenschaftliche Karriere hat zur Folge, dass der Zweck von Ausgründungen dann vornehmlich aus wissenschaftlicher Sicht interpretiert wird. Aus einer auch ökonomisch gesicherten Lage heraus werden Ausgründungen als zusätzliche Möglichkeit („Pipeline“) beschrieben, um zu prüfen, ob das, was man sich ausgedacht hat, auch tatsächlich funktioniert (praktische Validierung von Ideen). Eine weitere Perspektive professoraler Beschäftigungen mit Ausgründungen findet sich im Interesse daran, dass diese Art der Verwertung weitere Forschungsmittel generiert oder zumindest Nachwuchswissenschaftler mit Erwerbsmöglichkeiten versorgt. Häufig sind solche Ausgründunge auch ein Vehikel dafür, die vermeintlich bürokratischen Kontrollen kameralistischer Organisationen zu umgehen und im Drittmittelgeschäft über ein flexibles Instrument zu verfügen. Ausgründungen, an denen Hochschullehrer dauerhaft beteiligt bleiben, diese sogar zu einem Wechsel von der verbeamteten Professur zum Geschäftsführer oder Vorstandsmitglied motivieren, bleiben indes in Deutschland die Ausnahme.

Komplizierter stellt sich die Lage für den in Ausgründungen involvierten wissenschaftlichen Nachwuchs dar. Das

2 Es handelt sich hierbei u.a. um die Forschungsprojekte "Wissenschaftsunternehmer: Typus, Merkmale und Erfolgsbedingungen von akademischen Grenzgängern" (gefördert vom BMBF); "Evaluation von EXIST III" (gefördert vom BMWi) und "Production of Knowledge Revisited “ (PROKNOW), gefördert von der EU. Diese Studien wurden von der Forschungsgruppe „Wissenschaftspolitik“ am Wissenschaftszentrum Berlin für Sozialforschung in der Zeit zwischen 2004 und 2009 realisiert.

3 Die Auswahl der Probanten folgte dabei der Annahme, das man auf der Suche nach einer nachhaltigen Verknüpfung wissenschaftlicher und wirtschaftlicher Bezüge Ansätze einer wissenschaftlichen Sozialisation (Promotion) und eine mehr oder weniger kontinuierliche Präsenz ökonomischer Relevanzen (Unternehmensgründungen) sicherstellen muss. Die in den Abschnitten 3.2 bis 3.4 referierten Zitatausschnitte entstammen alle den aufgezeichneten Gesprächen. 
Gründungsmotiv ist dabei, „während und anstatt" einer ungesicherten wissenschaftlichen Karriere nach Alternativen zu suchen. Der Auslöser kann hierbei ganz verschiedener Art sein. Die ideelle und materielle Unterstützung bleibt aus, das Interesse an einer wissenschaftlichen Karriere erlahmt gegenüber alternativen Berufswegen oder man stößt während der Forschungsarbeit auf verwertungsrelevante Ergebnisse, die weitere berufliche Optionen eröffnen. Der „überwechselnde Nachwuchswissenschaftler" ist dabei aber immer mit einem potenziellen Abbruch der wissenschaftlichen Karriere und einer Neuorientierung in einem wirtschaftlich und durch Entwicklungsaufgaben bestimmten Feld konfrontiert. Für diese strategische Phase einer Karriere gibt es im deutschen Ausbildungswesen keine systematische Unterstützung. Während die wissenschaftlichen Fachgemeinschaften auch ein temporäres Verlassen der akademischen Karrierepfade unmittelbar mit Resonanzverlust bestrafen, fehlen für die neue Karriere als Unternehmer die Kenntnisse, die Kompetenzen und damit auch das $\mathrm{Zu}$ trauen. Überwechselnde Wissenschaftler sind im wahrsten Sinne „Emigranten“, sie verlassen ihr „Sicherheitsnetz“, ohne sich eines neuen Ankunftsorts bereits gewiss zu sein (Möll/Jacobsen 2010). Die Folge dieser berufsbiografisch äußerst riskanten Situation ist häufig, dass die Entscheidung, Wissenschaftler zu bleiben oder Unternehmer zu werden, verzögert und in der Schwebe gehalten wird. Ein Zustand, der von den klassischen Unterstützereinrichtungen wie den Transferstellen oder Karriereberatung völlig unterschätzt und fehl gedeutet wird (Egeln et al. 2010).

Bemerkungen der Probanten, sich in Deutschland doch lieber schnell für einen Weg zu entscheiden, finden hiermit ihre Begründung. Der in dieser Übergangsphase durchaus zu beobachtende Versuch, sowohl wissenschaftlich relevante Beiträge zu produzieren und parallel mit der Weiterentwicklung eines für die Unternehmensgründung notwendigen Produkts oder Verfahrens fortzufahren, findet in der Rigidität der Rollenerwartung seine Grenze.

\subsection{EXKLUSIVITÄT DER KERNROLLEN}

Bereits der Hinweis, Produktentwicklungen und Unternehmensgründungen benötigen zusätzliche Zeit und befördern daher eine klare berufsbiografische Ent- scheidung, verweist auf etwas Grundsätzliches. Wenn man zusätzliche Zeit benötigt, dann bedeutet das, dass die Produktion von wissenschaftlichen Beiträgen und von unternehmerisch verwertbaren Produkten zwei unterschiedliche, mithin schwer zu vereinbarende Prozesse sind, selbst wenn sie sich auf das gleiche Objekt richten mögen. Ausgründer machen regelmäßig die Erfahrung, dass das wissenschaftliche Basiselement Publikation sowie die dort verlangte Ausrichtung am Verstehen und Erklären von Zusammenhängen an Bedeutung verliert, sobald die unternehmerisch relevante Orientierung an funktionierenden und vermarktbaren Produkten wichtig wird:

„Also mir wär's vor meinem Verständnis als Wissenschaftler [...] lieber gewesen, dass ich noch mehr Zeit hätte, in Ruhe noch verschiedene andere Optionen auszuprobieren vor einem wissenschaftlichen Hintergrund, weil also das, was wir uns da überlegt haben und erfunden und als Patent gesichert haben, das funktioniert, aber wir verstehen eigentlich noch nicht gut genug warum, es tut's halt. Und das ist halt die pragmatische Herangehensweise von Firmenleuten oder sobald Investoren ins Gespräch kommen, da interessiert eigentlich nur, funktioniert's oder nicht, und das Verständnis, ob's funktioniert und warum, das juckt die gar nicht" (Interview).

Im Zuge der Ausgründung beginnt die Publikation ihre genuine Funktion zu verlieren, weil diese nicht der unmittelbare Geschäftszweck ist, sondern eher nebenbei erledigt wird oder „um zu dokumentieren, dass wir qualitativ hochwertige Arbeit hier im Unternehmen machen" (Interview). Wissenschaftliche Veröffentlichungen können auch im Unternehmensalltag als Qualitätsmaßstab dienen, der auch im Geschäftsleben in den Verhandlungen mit Kunden oder Banken mitunter eine gewisse Bedeutung erlangt. Allerdings stoßen junge wissenschaftlich sozialisierte Unternehmensgründer auch sehr schnell auf einen anderen Umstand, der eine Veröffentlichung von Arbeitsergebnissen unmöglich macht: „Generell publizieren wir nichts, weil, wenn wir etwas machen, dann ist es normalerweise direkt im Auftrag für Kunden und dann ist das natürlich mit einem non-disclosure-agreement verbunden" (Interview). Die strukturelle Entwertung wissenschaftlichen Publizierens resultiert jedoch nicht nur aus Interessengegensätzen. Denn die Entwicklung von funktionierenden und später marktgängigen Produkten baut auf bereits gesichertem technologischen Wissen auf und erfordert umfangreiche standardisierbare Testverfahren, die oftmals nicht mehr im Interesse der Wissenschaftler liegen, weil hier keine neuen und insbesondere originellen Erkenntnisse zu erwarten sind. „Es ist rein wissenschaftlich gesehen dann nicht mehr so interessant, sondern es ist ne reine Fleißarbeit, aber es ist natürlich interessant, ob das Medikament wirkt, aber man kriegt da wirklich jetzt keine neuen Erkenntnisse über irgendwelche Prozesse oder so" (Interview).

Diese Schwierigkeiten, die wissenschaftliche Erkenntnisarbeit mit unternehmerischer Arbeit zu vereinbaren, wie auch die immer wieder geforderte Entscheidungssituation führen dann dazu, dass Berufsrollen von den betreffenden Wissenschaftlern selbst strikt auf ihre jeweiligen Kernfunktionen zurückgebunden werden. In der Folge konstituieren beide Rollengefüge ein wechselseitiges Ausschlussverhältnis. Die Exklusivität der das Handeln orientierenden Zentralfunktionen zeigt sich dann sogar in den Zuschreibungen von persönlichen Eigenschaften und Fähigkeiten, die diese Rollenanforderungen ausfüllen können. Aus solchen Eigenschaftsbündeln ergeben sich prägnante Muster, die Karriereentscheidungen von Nachwuchswissenschaftlern anleiten oder auch als nachträglich angelegte Begründungsschablone Verwendung finden und sich damit gegenseitig zusätzlich verstärken. So begründet beispielsweise ein Ausgründer seinen beruflichen Wechsel zum Unternehmertum mit seiner unzureichenden Akribie: „Im Prinzip glaube ich, der Gründer ist nicht ein Forscher. Oder die Charaktere widersprechen sich wahrscheinlich so ein bisschen, weil ein Gründer, der darf nicht in jedem Bereich ins Detail gehen. Der Forscher muss in jedem Bereich ins Detail gehen. Wenn ich mich anschaue, ich hatte nie Spaß [...] die letzte Formel bis zum Ende auszurechnen“ (Interview). Umgekehrt werden auch gerne getroffene Personalentscheidungen mit den sich selbst verstärkenden exklusiven Rollenzuschreibungen legitimiert. Ein Professor verwendet eine ähnlich Unterscheidung zur Identifikation unternehmerischer Begabung: „Sie sind schneller, sicher. Sie haben nicht unbedingt die Tiefe, aber es gibt einfach Typen, die haben im Blick: Was könnt ich damit machen?" (Interview). 


\subsection{INSTABILES ROLLENMUSTER}

Wie aber gehen diejenigen, die an den Naht- und Übergangsstellen zwischen Wissenschaft und Wirtschaft an einer Überwindung bzw. an einer Verbindung von stabilen und auf Exklusivität beharrenden Berufsrollenverständnisse interessiert sind, damit um? Eine berufliche Existenz dazwischen oder in beiden Bereichen zu haben, erscheint gleichermaßen notwendig und dennoch praktisch unmöglich zu sein: „Ja, das sind zwei Dinge, die angelegt sein müssen. Erstens wissenschaftliche Neugier [...], also Spieltrieb, Neugier, visionäre Kraft, und zweitens praktische Umsetzungsfähigkeit, also auch die Neugier, mal Dinge fliegen zu sehen, fahren zu sehen, arbeiten zu sehen, die man selbst im Kopf entwickelt hat" (Interview). Auch die internationale Forschung zur Gestalt des idealerweise mit wissenschaftlicher Neugier und der Fähigkeit zum „Durchsetzen neuer Möglichkeiten auf wirtschaftlichem Gebiet" (Schumpeter 1928) ausgestatteten Wissenschaftsunternehmers, berichtet bei genauerer Betrachtung weniger von einer Zusammenführung dieser unterschiedlichen Bezüge in einer Person, als von Rollendoppelungen und Rollenkonflikten. Die Gegenüberstellung von „Academic Entrepreneur" und „Entrepreneurial Academic“ (Meyer 2003) oder von „Academics“ und „Pioneers“ (Shinn/Lamy 2006) bezeichnen nur den Sachverhalt, dass Unternehmensgründungen entweder aus der Sicht der Wissenschaftler- oder Unternehmerrolle betrachtet werden. Die Zwischenfiguren des „ideal-typical entrepreneurial scientist“ (Etzkowitz 1998), „Janus“ (Shinn/ Lamy 2006) oder „liminal entrepreneurial scientist" (Gulbrandsen 2005) sind durch einen beständigen Rollenwechsel und die Erfahrung gekennzeichnet, weder nur Wissenschaftler oder Unternehmer zu sein, beides nicht zugleich sein zu können, aber auch keine neue dritte berufliche Rolle und Identität entwickelt zu haben: „they neither identify with other basic researcher nor the entrepreneurial community" (Gulbrandsen 2005, S. 50).

Eine einfache Integration wissenschaftlicher und wirtschaftlicher Orientierungsmuster, Arbeitsweisen und Selbstverständnisse in einem stabilen beruflichen Status ist also sehr schwierig, von einer eigenen Profession eines „Wissenschaftsentrepreneurs" kann schon gar keine Rede sein. Die Gründe hierfür sind genannt: Die Integration der Primärrollen Wissenschaftler und Unternehmer ist zu anspruchsvoll gedacht, weil sie jeweils in sehr unterschiedlichen und rigorosen Referenzsystemen eingebunden sind, die direkt an die Kernfunktionen von Wissenschaft und Ökonomie anschließen, der Wissenschaftsunternehmer steht immer in Konkurrenz zu seinen Primärrollen. Es mangelt an Grenzübergängen oder Übergangsgebieten, in denen hybride Arbeitsund Referenzbereiche ihre Berechtigung erhalten und respektiert werden. Dem Wissenschaftsunternehmer stehen weder eine spezielle Wissensbasis, erfahrene Vorbilder noch die Möglichkeit zur Verfügung, sich in eine besondere Berufspraxis einzufinden. Wissenschaftsunternehmer zu sein, ist folglich eine riskante, individuelle und mit keinem kondensierten Erfahrungsschatz ausgestattete Tätigkeit.

\subsection{AUF DEM WEG ZU NEUEN BERUFSROLLEN}

Bei der Auswertung der empirischen Ergebnisse stößt man innerhalb dieser Grundspannungen aber auch immer wieder auf Lösungsversuche, die divergierenden Anspruchswelten doch in einem dauerhaften „dritten“ Weg zu integrieren. Aus diesen Versuchen lässt sich etwas über die Bedingungen lernen, wie sich auch unter diesen Umständen neue Berufsrollen herausbilden könnten. Diese Versuche sind zunächst allesamt durch eine doppelte Abgrenzung vom selbstgenügsamen Streben der Wissenschaft nach Erkenntnis und Deutung einerseits sowie vom primären Ziel ökonomischen Gewinns andererseits gekennzeichnet. Neue Identitäten können möglicherweise dann entstehen, wenn ein eigenständiger Tätigkeitsbereich definiert und offensiv besetzt werden kann (Abbott 1988). Wissenschaftsunternehmer müssten demnach darauf abzielen, eine eigene Perspektive zu reklamieren, statt sich zwischen den hermetisch abgeschlossenen Referenzbereichen zu positionieren und immer auf wissenschaftliche sowie wirtschaftliche Arbeitsinhalte gleichermaßen zu beharren. Diese Voraussetzung erfüllen Versuche, denen es gelingt, ein eigenes Berufsziel jenseits beider Rollen zu formulieren und zur Grundlage ihres Handelns zu machen. Eine besonders oft anzutreffende Strategie ist die Orientierung am Berufsbild des „Ingenieurs“. Im Mittelpunkt des professionellen Interesses steht in den Worten eines
Probanten die Arbeit mit „ganz einfachen Modellen“ und an „kleinen“ und „reifen“ Problemen, die nicht erklären wollen und können „was die Welt im Innersten zusammenhält". Auch die Durchsetzung am Markt muss nicht in Eigenregie erfolgen: „Wir versuchen, das so schnell wie möglich los zu werden“. Ingenieure haben bereits ein kollektiv geteiltes Verständnis, dass unabhängig von der jeweiligen Fachdisziplin in einem Berufsbild stabilisiert ist. Diese Orientierung scheint in den Grenzbereichen ein hohes Maß an beruflicher Identität zu vermitteln. Zwar erlebte diese Berufsgattung oft genug prekäre Situationen, entweder als Agenten des Kapitels oder als Technokraten kritisiert zu werden. Aber mit einem eigenständigen kanonisierten Methodenwissen und einer Ausrichtung der Tätigkeit an Kriterien eigener Art hat sich der Ingenieur als ein eigenständiger Beruf zwischen Wirtschaft und Wissenschaft behaupten können (Neef/Pelz 1997).

Die zweite Variante, gegenüber Wissenschaft und Ökonomie zu einem eigenen Berufsbild zu kommen, findet sich bei einem Teil der medizinischen Biotechnologen. Berufsziel ist hier, mit neuen Medikamenten „dem Menschen zugute“ zu kommen. Wie bei den klassischen Professionen der Medizin oder des Rechts rückt ein gesellschaftlich relevanter Eigenwert (Gesundheit oder Recht) ins Zentrum, der eine eigensinnige Handlungsorientierung sichert und zur Abgrenzung von einer rein erkenntnisgesteuerten Wissenschaft führt: „Mein Ziel war nicht, zu erforschen, ob die Schnecke sich links oder rechts rumdreht, was ja sicher auch interessant ist, [...] ich wollte was tun, was dann am Ende der Gesellschaft zugute kommt" (Interview). Das Finanzierungsproblem und -risiko hierfür muss dann auch gesellschaftlich oder gegebenenfalls privatwirtschaftlich getragen werden, jedenfalls ist es ebenfalls nicht Teil des eigenen Berufsverständnisses: „Wir brauchen jetzt erstmal Geld und ihr müsst uns glauben, dass das, was wir hier vorhaben, werthaltig ist"(Interview). Diese noch sehr heterogene Gruppe der Biotechnologen hat in Deutschland erst seit gut zehn Jahren begonnen, eigene Curricula zu entwickeln und diese besonders an Fachhochschulen zu etablieren. Doch bestärkt und unterstützt von sehr mächtigen Fachgesellschaften wie der Gesellschaft für chemische Technik und Biotechnologie (DECHEMA) und angetrieben durch eine Vielzahl von Bundes- und 
Landesförderungen kristallisieren sich hier mittlerweile eigenständige Orientierungen und Profilbildung zwischen erkenntnisgetriebener Forschung und wirtschaftlichem Gewinnstreben heraus.

Ein weiterer Versuch, zugleich „Forschender, Mediziner und Unternehmer" $\mathrm{zu}$ sein und alle drei Rollen gleichermaßen in einem Beruf zu vereinbaren, kann dann gelingen, wenn man sich von einer auf Dauer angelegten Entscheidung zwischen diesen verabschiedet und stattdessen ein eigenständiges Berufsziel formuliert. Als „Berater“ für die Überführung wissenschaftlicher Erkenntnisse in ökonomisch verwertbare Produkte benötigt man zwar einen fundierten Sachverstand in allen drei Segmenten. Dieser bildet aber nur den Ausgangspunkt, um dann als „Mittler“, „Bindeglied“ und „Figur dann in der Mitte" eine selbstständige Berufsrolle und Berufstätigkeit auszubilden. Die Grundspannungen, gleichermaßen in allen Referenzbereichen zu Hause zu sein, kann man durch die Definition eines jeweils nur zeitlich begrenzten Engagements offenkundig sehr produktiv auflösen. Jedenfalls ist eine Reihe von ambitionierten Naturwissenschaftlern, die sich zwischenzeitlich in Spin-offs oder Start-ups versucht haben, mittlerweile als Berater unterwegs und beginnt - ebenfalls unterstützt durch verschiedene Förderprogramme - an einem konsolidierten Tätigkeitsbild zu arbeiten.

Ingenieure, Biotechnologen und Berater sind Varianten, die sich durch die zweifache Abgrenzung definieren. Die Gemeinsamkeiten aller drei Versuche zur Bildung eines eigenständigen Berufsverständnisses liegen darin, weder das wissenschaftliche Referenzsystem als eine auf Dauer existierende Orientierung zu akzeptieren, noch den Sinn im unmittelbaren wirtschaftlichen Verwerten der Ergebnisse zu finden. Erfolgreich scheinen diese praktisch gelebten Versuche des „Wissenschaftsunternehmers" auch deshalb zu sein, weil an z.T. bereits vorgelebte Berufsorientierungen angeknüpft werden kann und keine völlig neuen Bilder generiert werden müssen.

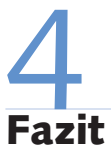

Was können sozialwissenschaftliche Gesellschaftsdiagnostiker und politische Gestalter gleichermaßen aus unserem Beispiel
"Wissenschaftsunternehmer" lernen? Aus den Ergebnissen der empirischen Studien scheinen uns drei Punkte für eine allgemeine Diskussion bemerkenswert:

(1) In der Debatte um angemessene Bedingungen für eine schnelle Umsetzung wissenschaftlicher Erkenntnisse in marktfähige Produkte und Dienstleistung fehlt ein wichtiges Übersetzungsglied. Die Funktionsbereiche Wissenschaft und Wirtschaft haben keine natürlichen oder automatischen Verbindungs- oder Übergangsbereiche. Diese müssen immer aufs Neue etabliert und stabilisiert werden. Die auf den ersten Blick als unübersichtliche Gemengelage erscheinende Transferlandschaft löst sich bei einem zweiten Blick in ganz unterschiedliche Seiensbereiche auf, die noch immer durch äußerst stabile Identitäten mit scharfen Kanten charakterisiert sind und die wie Demarkationslinien wirken. Übergänge stellen eher die Ausnahme als den Regelfall dar. Von einer fortschreitenden „Entgrenzung“ und „Vermischung“ kann deshalb nicht die Rede sein.

(2) Für die Bewegung in diesen Räumen fehlt es daher auch an stabilen Orientierungen und Leitsystemen. Der für das deutsche Innovationssystem so typisch kategorische Zwang, sich entweder als Wissenschaftler oder als Unternehmer $\mathrm{zu}$ verstehen und $\mathrm{zu}$ behaupten, lässt kaum Zwischenspiele möglich werden. Zugleich verläuft die Überführung von wissenschaftlicher Erkenntnisarbeit in marktfähige Produkte aber nicht linear, sondern rekursiv, und kann deshalb auch nicht im Rahmen dieser stabilen Primärrollen sequenziell abgearbeitet und dann übergeben werden. So lange es für diese Grenz- und Übergangsbereiche keine neuen Berufsidentitäten, eingeschränkten Tätigkeitsspektren und eingeschliffenen Karrierewege gibt, bleibt das berufsbiografische Projekt, Wissenschaftsunternehmer zu werden, ein äußerst riskantes und höchst individuelles Vorhaben, das aus guten Gründen nur wenige eingehen.

(3) Innerhalb der Diskussion um die Innovations- und Wissenschaftspolitik wurde der Frage, „wer bin ich?“" viel zu wenig Aufmerksamkeit geschenkt. Wissenschaftsunternehmer als gedachte Hybride, die für den Übergang von Wissenschaft zur Wirtschaft als Schlüsselfiguren gelten, kommen als natürliche Wesen im deutschen Wissenschaftssystem nicht vor; die jeweils vorherrschenden Berufsrollen beharren auf einem so exklusiven Identitätskonzept, dass eine wechselseitige Vermischung praktisch nicht möglich, aber wissenschaftspolitisch auch nicht zwingend geboten ist. Gerade in den Übergangszonen bedarf es der Herausbildung eigener beruflicher Orientierungen, um nicht zwischen den heterogenen Ansprüchen der Wissenschaftler- und Unternehmerrolle zerrieben zu werden. Erst durch den Aufbau eines eigenen Referenzbereichs können sich Arbeitsweisen langfristig stabilisieren und neue berufliche Identitäten generiert werden. Und hier liegen sicherlich die größten politischen Gestaltungsaufgaben versteckt; wie kann man zwischen den dominanten Berufsbildern neue, attraktive Perspektiven entstehen lassen, die sich nicht zufällig oder über Umwege zwischen den Demarkationslinien herausbilden, sondern für die es gangbare, wechselseitig akzeptierte und respektierte Berufswege eigener Art gibt?

Wenn also die oben zitierte Annahme richtig ist, dass für notwendige Neuerungen die Verbindung wissenschaftlicher Erkenntnisarbeit mit unternehmerischem Geschäftssinn eine wichtige Voraussetzung ist, dann kann dies offenkundig nur gelingen, wenn hierfür ein dritter Weg einer professionellen Entwicklung gefunden werden kann. Zu klären wäre dann, wer die notwendigen Akteure wären und welche Instrumente man zur Realisierung bräuchte. Sicherlich bietet der sich zurzeit in der deutschen Hochschullandschaft abzeichnende Differenzierungsprozess hier interessante Gestaltungsperspektiven. Man könnte vermuten, dass die zunehmende Anforderung an Universitäten, neben Forschung und Lehre auch für außerakademische Arbeitsmärkte auszubilden, ein geeignetes Feld für die Entwicklung dieser Berufsbilder sein könnte. Selbstredend müssten hierbei wissenschaftsbezogene und berufsbezogene Ausbildungsgänge zu ihrem eigenen Recht kommen und dabei durch eine stärkere institutionelle Ausdifferenzierung innerhalb oder außerhalb der Universität unterstützt werden. 
Abbott, A. (1988): The System of Professions. An Essay on the Division of Expert Labor, Chicago/London

Borcherding, A./Torka, M. (2009): Akademische Grenzgänger. Wissenschaftsunternehmer haben noch keine feste Rolle gefunden, in: WZB-

Mitteilungen 123 , S. 26-29

Braun-Thürmann, H./Knie, A./Simon, D. (2010): Unternehmen Wissenschaft. Ausgründungen als Grenzüberschreitungen akademischer Forschung, Bielefeld

Bush, V. (1945): Science, the endless Frontier. A Report to the President on a Program for Postwar Scientific Research, July, Washington Egeln, J./Dinges, M./Knie, A. (et al.) (2010): Evaluation des Existenzgründungsprogramms EXIST III, Baden Baden

Etzkowitz, H. (1998): The Norms of Entrepreneurial Science: Cognitive Effects of the New University-Industry Linkages, in: Research Policy 8, S. $823-833$

Gibbons, M./Limoges, C./Nowotny, H.;/Schwartzman, S./Scott, P./ Trow, M. (1994): The new production of knowledge: the dynamics of science and research in contemporary societies, London

Gülker, S./Knie, A./Simon, D. (2009): Bachelor und Bologna: Warum die Reform reformiert werden muss, in: WZBrief Bildung vom 10. November

Gulbrandsen, M. (2005): „But Peter's in it for the money " the liminality of entrepreneurial scientists, in: VEST 1-2.05, S. 49-75

Knie, A./Braun-Thürmann, H. (2008): Katalysator des Wandels: Die Wirkung der Exzellenzinitiative auf das Verhältnis von Wirtschaft und Wissenschaften, in: Hornbostel, St./Simon, D./Heise, S. (Hrsg.): Exzellente Wissenschaft: Das Problem, der Diskurs, das Programm und die Folgen, iFQ-Working Paper 4, Oktober, S. 81-92

Loer, Th. (2006): Zum Unternehmerhabitus. Eine kultursoziologische Bestimmung im Hinblick auf Schumpeter, Karlsruhe
Meyer, M. (2003): Academic entrepreneurs or entrepreneurial academics? Research-based venture and public support mechanisms, in: R\&D Management 2, S. 107-115

Möll, G./Jacobsen, H. (2010): Wissenschaftler zwischen Akademie und Ökonomie. Zur Herausbildung neuer Formen wissenschaftlicher Beruflichkeit, in: Braun-Thürmann, H./Knie, A./Simon, D.: Unternehmen Wissenschaft. Ausgründungen als Grenzüberschreitungen akademischer Forschung, Bielefeld, S. 75-116

Münch, R. (2006): Die akademische Elite. Zur sozialen Konstruktion wissenschaftlicher Exzellenz, Frankfurt a.M.

Musselin, Ch. (2007): The Transformation of Academic Work: Facts and Analysis, in: Center for Studies in Higher Education, Research \& Occasional Paper Series: CSHE.4.07

Neef, W./Pelz, Th. (Hrsg.) (1997): Ingenieurinnen und Ingenieure für die Zukunft: Aktuelle Entwicklungen von Ingenieurarbeit und Ingenieurausbildung, Berlin

Rammert, W. (1988): Das Innovationsdilemma, Wiesbaden

Schumpeter, J. A. (1928): Unternehmer, in: Ders.: Beiträge zur Sozialökonomik, Wien, Köln, Graz, hrsg. von Böhm, S. (1987), S. 137-157

Shinn, T./Lamy, E. (2006): Paths of commercial knowledge: Forms and consequences of university-enterprise synergy in scientist-sponsored firms, in: Research Policy 10, S. 1465-1476

Simon, D./Knie, A./Hornbostel, St. (Hrsg.) (2010): Handbuch Wissenschaftspolitik, Wiesbaden

Slaughter, Sh./Leslie, L.L. (1997): Academic Capitalism: Politics, Policies, and the Entrepreneurial University, Baltimore

Torka, M./Borcherding, A. (2008): Wissenschaftsunternehmer als Beruf? Berufs- und professionssoziologische Überlegungen vor dem Hintergrund aktueller (Ent-) Differenzierungsphänomene der Wissenschaft, Schwerpunkt III 2008-601 